\title{
基于中性铂炔基元的超分子有机金属凝胶研究进展
}

\author{
范文佳陈丽君* 杨海波* \\ (华东师范大学化学系 上海市绿色化学与化工过程绿色化重点实验室 上海 200062)
}

\begin{abstract}
摘要 作为一类经典的软物质材料, 超分子凝胶在催化、化学传感、药物释放等许多领域展示了广泛的应用前景. 最 近人们研究发现, 在基元砌块中引入金属特别是过渡金属之后, 所形成的有机金属凝胶在光电材料、催化、磁性等方 面具有独特的物理化学性质. 近年来，电中性铂炔基元因其独特的线性结构、易合成及丰富的光物理性质在超分子化 学及材料科学中被广泛应用. 总结了基于中性铂炔基元的超分子有机金属凝胶的构筑及其功能研究进展, 主要包括以 苯撑共轭结构为核心骨架的铂炔有机金属凝胶以及官能团修饰的铂炔有机金属凝胶.
\end{abstract}

关链词 超分子化学; 自组装; 有机金属凝胶; 铂炔基元; $\pi$-共轭体系

\section{Supramolecular Organometallic Gels Based on Neutral Platinum-Acetylide Moiety}

\author{
Fan, Wenjia Chen, Lijun* Yang, Haibo* \\ (Shanghai Key Laboratory of Green Chemistry and Chemical Processes, Department of Chemistry, \\ East China Normal University, Shanghai 200062)
}

\begin{abstract}
Supramolecular gels, as a family of classical soft materials, have received considerable attention because of their wide potential applications in catalysis, chemical sensing, drug delivery carrier, and many other areas. Recently, it was found that the introduction of metal moieties, especially the transition metal moieties into the gelator allows for the additional scope for tuning gel properties such as catalytic activity, magnetism, and photo- and electrochemistry. Neutral platinum-acetylide complex have been investigated in many respects from supramolecular chemistry to materials science during the past two decades because of their attractive properties such as well-defined linear geometry, synthetic accessibility and intriguing photoproperties. In this review article, we summarize the recent results in the investigation of supramolecular organometallic gels based on neutral platinum-acetylide moieties. These systems are classified based on the skeleton of the gelator building blocks and divided into two main parts, conjugated phenylene cored platinum-acetylide derivatives as well as other platinum-acetylide derivatives containing various functional groups.

Keywords supramolecular chemistry; self-assembly; organometallic gels; platinum-acetylide moiety; $\pi$-conjugated system
\end{abstract}

凝胶是一种常见的物质凝聚状态, 通常被界定为一 种介于固体和液体之间的准固态物质(软物质). 1974 年, Flory 等 ${ }^{[1]}$ 为凝胶给出了一个被广为接受的定义: “凝胶 具有连续的结构, 在分析过程中性质稳定, 而它的流变 学性质与固体相似” . 这种固态结构和液相共存的状态 赋予凝胶独特的弹性特征并且不同于传统意义上的固 体和液体材料. 比如发胶、隐形眼镜、果冻等都是在日 常生活中广泛应用的凝胶. 近年来, 随着超分子化学的 发展, 超分子凝胶因其与传统的聚合物凝胶在结构和性
质上的差异受到化学家们的广泛关注. 超分子凝胶是指 低分子量凝胶因子(Gelator)与溶剂分子间通过各种非共 价弱相互作用力，例如配位键、氢键、范德华力、 $\pi-\pi$ 堆积作用、疏水-亲水作用等, 自组装形成各种有序结 构, 并进一步通过相互间弱相互作用自组装形成三维网 络结构, 从而阻止了溶剂分子的自由流动, 呈现一种半 固体的状态 ${ }^{[2,3]}$. 超分子凝胶又被称作低分子量凝胶 (LMOG, Low Molecular Mass Gel) ${ }^{[4 \sim 6]}$. 与传统的聚合物 凝胶相比, LMOG 通常具有加热溶解性好、溶胶一凝胶可

*E-mail: hbyang@chem.ecnu.edu.cn; 52130606022@ecnu.cn

Received October 31, 2014; revised December 2, 2014; published online January 7, 2015.

Project supported by the Key Basic Research Project of Shanghai Science and Technology Commission (No. 13JC1402200) and the Outstanding Doctoral Dissertation Cultivation Plan of Action (No. PY2014011).

上海市科委基础重点(No. 13JC1402200)与优秀博士学位论文培育行动计划(No. PY2014011)资助项目. 
发生热可逆相转变的特点 ${ }^{[7 ~ 9]}$. 值得一提的是, 通过对 凝胶因子的合理设计, 可以实现凝胶对 $\mathrm{pH}^{[10]}$ 、温 度

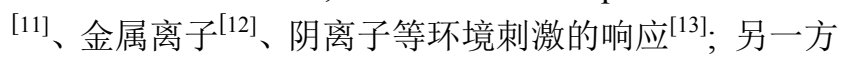
面, 将各类不同的功能性官能团引入凝胶因子中, 也可 以实现功能化的超分子凝胶的构筑 ${ }^{[14,15]}$.

\section{1 超分子有机金属凝胶}

超分子有机金属凝胶(Supramolecular Organometallic Gel)通常指含有金属的超分子凝胶体系, 金属的引入 通常为所形成凝胶带来诸如催化、光电活性、磁性能等 一系列新的物理化学性质 ${ }^{[16,17]}$. 利用金属-配体的多样 性, 以及金属与配体之间的弱相互作用可调节性, 可以 更容易地控制超分子自组装过程, 从而影响凝胶的特 性. 值得注意的是, 凝胶因子中的金属离子, 特别是过 渡金属, 会在成胶过程中影响其凝胶因子的自聚集模 式, 并允许其更大程度地调整凝胶性质.

近年来, $\mathrm{Co}(\mathrm{II}) 、 \mathrm{Ag}(\mathrm{I}) 、 \mathrm{Au}(\mathrm{I}) 、 \mathrm{Pd}(\mathrm{II})$ 等一系列过渡 金属元素均被超分子化学家们用来构筑超分子有机金 属凝胶 ${ }^{[18]}$. 例如, 2002 年, 徐斌课题组 ${ }^{[19 \sim 21]}$ 利用衍生化 的杯 [4]芳烃和 $\left[\mathrm{Pd}(\mathrm{en})\left(\mathrm{H}_{2} \mathrm{O}\right)_{2}\right]\left(\mathrm{NO}_{3}\right)_{2}$ 成功构筑了一例两 组分超分子凝胶. 他们研究发现, 所形成的凝胶的稳定 性随着有机金属凝胶因子浓度的增加而显著提高. 同 时, 通过升高环境温度, 加入疏水性或亲水性的溶剂, 发现在合适的环境条件下该凝胶的宏观形貌得以保持, 并且能够相对较长时间稳定存在. 这一实验现象证明了 延长凝胶因子的交联度会大幅提升有机金属凝胶的稳 定性. 同时, Jung 课题组 ${ }^{[22]}$ 成功构筑了一例基于卟啉衍 生物的凝胶. 卟啉衍生物单体在 $\operatorname{Pd}(\mathrm{II})$ 的存在下可以通 过超声波和温度的控制, 实现溶液相一凝胶相两者之间 的转化. 在 $\operatorname{Pd}(\mathrm{II})$ 的存在下, 凝胶是在乙腈中通过超声 波诱导形成的. 而利用其他的外部刺激, 如剧烈振动或 微波辐射, 并不能诱导凝胶的形成. Uozumi 课题组 ${ }^{[23]}$ 也 报道了一类基于金属 $\mathrm{Pd}(\mathrm{II})$ 有机金属凝胶, 利用具有 $C_{3}$ 对称性的衍生化的三嗪环多齿配体, 通过加入 $\left[\mathrm{Pd}\left(\mathrm{CH}_{3} \mathrm{CN}\right)_{2} \mathrm{Cl}_{2}\right]$ 实现 $\mathrm{Pd}(\mathrm{II})$ 与有机小分子配体的配位络 合, 成功构筑了具有凝胶相 - 溶液相宏观变化的体系. 2005 年, Aida 课题组 ${ }^{[24]}$ 报道了一例基于 $\mathrm{Au}(\mathrm{I})$ 的超分子 有机金属凝胶, 主要利用 $\mathrm{Au}$ 和咪唑衍生物的配位作用 形成三角形的金属络合物, 同时通过外围长碳链的疏水 作用等弱相互作用成功制备了一例发光有机金属凝胶. 凝胶所产生荧光的颜色可由温度刺激、化学刺激等手段 进行调控. 与溶液相不同的是, 其凝胶相在紫外光激发 时会发出红色荧光; 当向体系中加入银离子(AgOTf)时, 凝胶相的荧光由红色转为蓝色; 当加热银离子掺杂的凝 胶后, 可得到发绿色荧光的溶液. Omary 课题组 ${ }^{[25]}$ 利用
Au-P 之间的弱相互作用, 成功构筑了一例具有高荧光 强度的水凝胶, 金属 $\mathrm{Au}$ 的引入提高了该类凝胶的苂光 强度.

铂(II) 是一类以平面四边形配位为主的过渡金属, 在过去的 20 年里, 由于其丰富的光物理和光化学性质, 有机金属铂配合物引起了超分子化学家们的广泛兴趣, 因此金属铂也常被用于超分子有机金属凝胶的构筑. 通 常, 铂炔化合物可以按照电性的不同分为两种类型, 带 电荷铂炔化合物和中性铂炔化合物. 带电荷的铂炔体系 还可以进一步分为阳离子和阴离子型. 阳离子型的铂乙 炔配合物主要包括铂与三联吡啶衍生物或其他一些类 似的 N-掺杂的三齿配体形成的带正电荷的配合物. 铂 乙炔基元的存在可以提高阳离子铂乙炔配合物的溶解 度, 从而有利于研究溶液中的凝胶因子的光物理和光化 学性质 ${ }^{[26-29]}$.

值得一提的是, 除了引入的有机配体所产生的常见 的非共价相互作用，单向的金属一金属相互作用(包括 $\mathrm{d}^{8}-\mathrm{d}^{8}$ 和 $\mathrm{d}^{10}-\mathrm{d}^{10}$ 的相互作用)也是有机金属凝胶的形成的 驱动力之一, 同时也往往伴随有趣的光物理和光化学现 象产生 ${ }^{[30]}$. 任咏华课题组 ${ }^{[18,31,32]}$ 报道了一系列基于 Pt-Pt 相互作用和 $\pi-\pi$ 堆积相互作用的超分子有机金属凝胶, 并对其光电性能进行了十分系统与详尽的研究. 例如, 2009 年, 该课题组 ${ }^{[3]}$ 利用长链烷基修饰的铂炔基元与 三联吡啶基团定向键合构筑了一例透明的有机金属凝 胶. 三联吡啶、苯并咪唑等含有不饱和氮杂环的配位基 团，一方面能够以其强配位能力和重金属离子进行定向 键合形成稳定配位键进而构筑相应的有机金属凝胶因 子; 另一方面，通过金属-金属之间的电荷转移作用可 以实现对凝胶的改性. 例如, 随着温度的升高, 铂一铂相 互作用被抑制, 故而深紫色的有机金属凝胶转变为橙色 溶液. 同时, 该有机金属凝胶的稳定性与 MMLCT(金 属一金属-配体的电荷转移)发射能量具有良好的相关性, 较低的 MMLCT 发射能量, 更强的铂一铂的相互作用可 以使三联吡啶铂炔凝胶因子在 DMSO 形成更稳定的凝 胶 ${ }^{[34,35]}$. Ziessel 课题组 ${ }^{[36]}$ 也成功制备了由铂(II)金属与 3,5-二酰胺基三联吡啶衍生物形成的凝胶因子, 研究表 明在聚集态时光谱同样显示了吸收和发射能量的明显 红移, 这种显著的能量转移同样是由于铂一铂相互作用 产生的. 当以正癸烷作为溶剂时, 随着溶液中凝胶因子 浓度的增加，可以在室温下形成深绿色的超分子有机金 属凝胶. 通过升高温度, 可以将凝胶相转变为红色的溶 液相.

中性铂炔基元因其独特的线性结构、易合成性及丰 富的物理化学性质在超分子化学及材料科学中被广泛 应用. 近年来, 大量关于线性中性铂炔基元在共轭有机 
金属聚合物及非线性光学材料等领域的文章及综述已 经被报道. 实际上，与带正电荷的铂炔基元类似，中性 的铂炔基元同样被广泛应用于超分子有机金属凝胶的 构筑中. 然而关于线性中性铂炔基元在超分子有机金属 凝胶中的研究目前还没有专门的综述. 作为基于带电荷 铂炔基元有机金属凝胶相关综述的补充 ${ }^{[18]}$, 本文将总 结近几年来基于中性铂炔基元的超分子有机金属凝胶 的研究进展. 按照核心骨架的不同, 本文将分为以下两 部分进行介绍: (1)以苯撑共轭结构为核心骨架的铂炔衍 生物凝胶; (2)官能团修饰的铂炔衍生物凝胶.

\section{1 以苯撑共轭结构为核心骨架的铂炔衍生物凝胶}

中性铂乙炔基元具有典型的铂(II)平面四边形这一 配位特征, 常见的为反式一铂 $\left[\mathrm{Pt}\left(\mathrm{PR}_{3}\right)_{2}\left(\mathrm{C} \equiv \mathrm{CR}^{\prime}\right)_{2}\right]$ 结构, 在 $\mathrm{C} \equiv \mathrm{CR}^{\prime}$ 中最为常见的则是 $\mathrm{R}^{\prime}$ 为芳基的结构. 中性铂乙 炔基元由于易于合成和衍生化, 同时又具有丰富的光学 特性, 因此已经成为高效构筑复杂、结构多样的超分子 体系的重要基元砌块 ${ }^{[37]}$. 在这些复杂的中性铂炔基元 中, 乙炔基和以三乙基膦作为膦配体形成的 $\mathrm{Pt}(\mathrm{II})$ 核心 骨架, 在许多常见的有机溶剂中均有较高溶解度. 高溶 解度是高产率制备和高效率纯化最终产物的必要条件. 近年来已有多例以苯撑共轭结构为核心骨架的铂炔衍 生物作为凝胶因子的报道.

2008 年, Schanze 等 ${ }^{[38}$ 报道了第一例基于苯撑共轭 结构为核心骨架的铂炔衍生物的超分子有机金属凝胶. 他们选用了 3 种不同的核心骨架结构的铂炔衍生物对其 成胶性能及光学性能进行了详细的研究(图 1). 铂炔基 元使有机金属骨架处于直线型有序排列, 同时末端芳环 上长链烷烃的引入为凝胶因子提供了疏水作用, 使凝胶
因子能够在癸烷中通过 $\pi-\pi$ 堆积及疏水作用聚集形成凝 胶. 电镜研究表明所成凝胶均表现为经典的纤维状微观 形貌，而对于含有手性基元的化合物 3 则表现为螺旋性 纤维. 光谱研究发现, 在凝胶相中可以观测到能量转移 现象, 并且这种现象仅在低聚物聚集体的混合物中存 在. 例如, 能量转移可发生在以正十二烷作为溶液的混 合交联体系中，而在氯仿溶液中因为 1 和 2 无法形成凝 胶，没有观察到能量转移现象. 更重要的是，进一步光 物理性质研究表明，三重态能量传递在凝胶相中是比较 有效的, 即使聚集体在很的低浓度条件下 $(1 \sim 2 \mathrm{~mol} \%)$, 能量传递也可以发生. 这也是第一例在超分子聚集体中 系统捕获三线态激子扩散的例子. 虽然三重态能量传递 的研究在分子晶体中已有一些研究和报道, 但是三重态 能量和激子在聚集体和层层之间的传递却鲜有报 道 ${ }^{[39,41]}$. 该工作在含有中性铂炔基元的超分子聚集体层 面上研究并探讨了三重态和能量转移的性质, 详细研究 了超分子聚集体中三重态激子的行为, 例如, 激发三重 态的产生、三重态激子的扩散和三重态一三重态能量传 递.

2013 年, 汪峰课题组 ${ }^{[42]}$ 报道了一例以棒状共轭苯 撑结构作为核心骨架的中性铂炔凝胶因子. 他们在凝胶 因子中引入了具有氢键作用位点的酰胺基元以提供额 外的弱相互作用力(图 2). 这类有酰胺基元修饰的铂炔 基元在室温下, 在常见有机溶剂中, 如正庚烷、正己烷 等, 可以形成稳定凝胶. 该课题组利用紫外吸收光谱研 究发现正是酰胺基团的引入导致了 $\mathrm{J}$ 型聚集体的形成, 从而证明了在凝胶形成过程中氢键的重要作用. 此外,
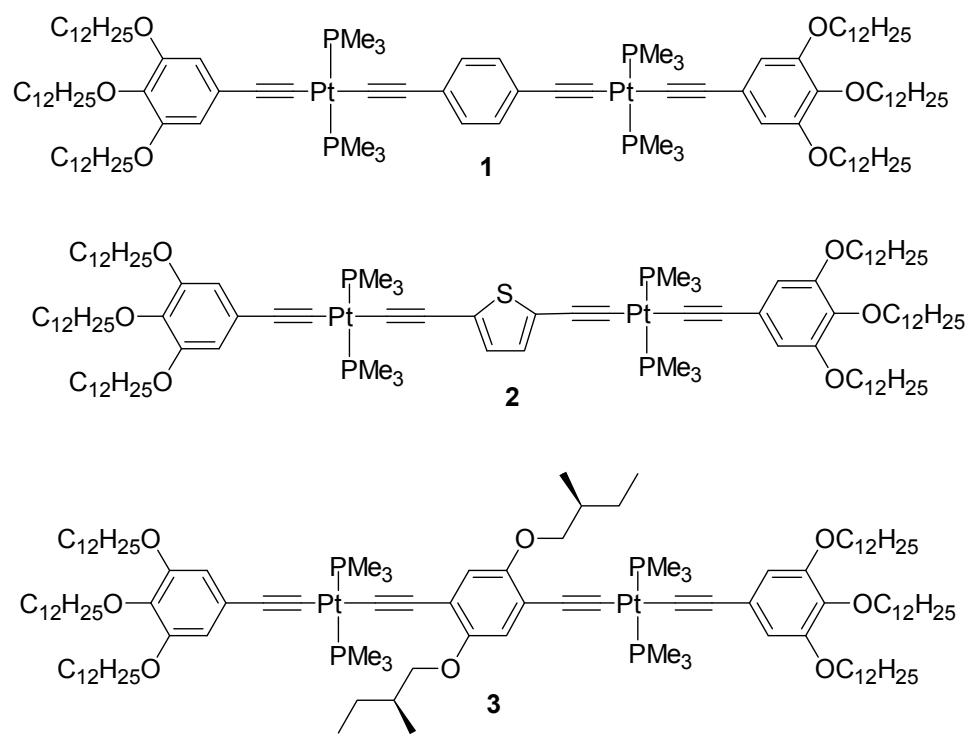

图 1 化合物 1,2 和 3 的结构

Figure 1 Chemical structures of compounds 1, 2 and $\mathbf{3}$ 
他们利用核磁氢谱对驱动聚集的 $\pi-\pi$ 堆积作用进行研 究, 特别是通过对协同作用机制的详细机理研究, 很好 地解释了一维超分子螺旋聚合物的形成.

基于铂炔基元的有机超分子凝胶的有效构筑不仅 依赖线性凝胶因子，具有其他形状与空间取向的苯撑铂 炔衍生物同样可以形成稳定的超分子凝胶. 2011 年, 杨 海波课题组 ${ }^{[43]}$ 设计合成一系列具有不同形状和空间取 向的中性铂炔共轭体系, 详细研究了凝胶因子的空间取 向对其成胶性能的影响. 研究发现具有矩形空间取向的 凝胶因子展现出很强的成胶能力 (图 3). 在多芳环体系 的 $\pi-\pi$ 堆积作用与长链烷烃的疏水作用驱动下, 凝胶因 子 6 和 7 可以在常见有机溶剂中以很低的临界凝胶浓度 值(CGC)形成稳定凝胶. 例如化合物 6 和 7 在正辛烷中 的 CGC 值分别为 2.0 和 $5.8 \mathrm{mg} / \mathrm{mL}$, 换言之, 这类凝胶 因子 6 和 7 可以在溶液中分别捕获 $2.42 \times 10^{4}$ 和 $0.35 \times$ $10^{4}$ 个溶剂分子. 对比化合物 6 和 7 的成胶能力, 研究发 现通过改变温度, 化合物 7 更倾向于形成溶液, 而化合 物 6 所形成的凝胶则具有相对较好的热力学稳定性. 这 一事实证明改变长链烷烃的长度可以作为调节凝胶性 质的一种重要手段. 另外有意思的是, 化合物 6 与 7 在 溶液相与凝胶相时呈现出完全不同的荧光性质, 而这种
荧光性质的差异可以通过样品的浓度变化或者外界温 度变化诱导其聚集形态改变来进行调控.

星状与树枝状 $\pi$-共轭分子通常表现出较好的导电 性和光学性能, 已被广泛用于构建有机功能材料和纳米 材料. 然而基于中性铂炔基元的星状与树枝状 $\pi$-共轭体 系的有机超分子凝胶的研究相对较少。因此, 2013 年, 杨海波课题组 ${ }^{[44 ~ 46]}$ 报道了一系列结构更为复杂的基于 大 $\pi$-共轭体系的星状与树枝状中性铂炔衍生物. 他们 ${ }^{[47]}$ 设计合成了一系列外围由长链烷烃修饰的含有铂炔基 元的树枝状和星射状化合物. 在这类新型的星射状凝胶 因子中, 烷基链与酰胺基团的存在使分子能够利用疏水 作用及氢键作用, 有序定向的聚集形成规整纳米结构. 光谱研究发现了该类树枝状或星射状化合物具有较丰 富的光物理性质. 通过对两类凝胶因子的光谱对比发 现，由于配合物 8a $\sim 8 b$ 具有更大的 $\pi$-共轭体系，其发射 相较于化合物 9a $\sim 9 b$ 有约 $100 \mathrm{~nm}$ 的红移. 同样通过对 比浓度的紫外一一荧光吸收光谱分析, 不难发现, 光谱 的浓度依赖性的变化可能是由于在高浓度溶液中邻近 的分子更倾向于发生分子间 $\pi-\pi$ 相互作用导致的. 另外, 众所周知，对一个长程有序的聚集体的形貌进行精确控 制是纳米科学和技术的重要研究内容, 因为这对器件的

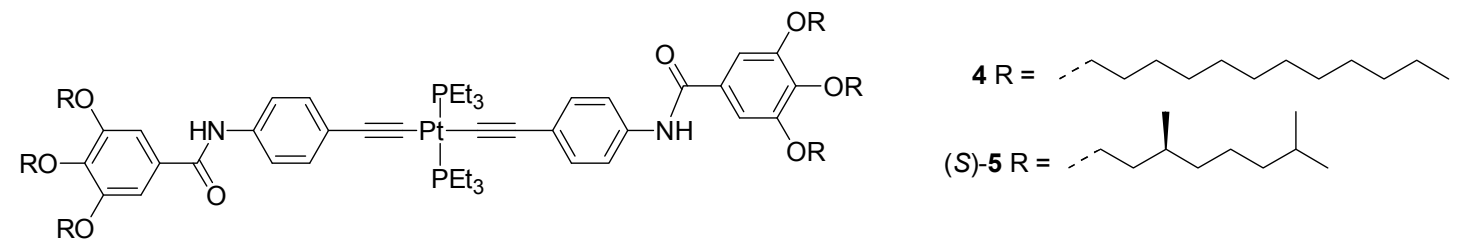

图 2 化合物 $\mathbf{4}$ 的结构

Figure 2 Chemical structure of compound 4

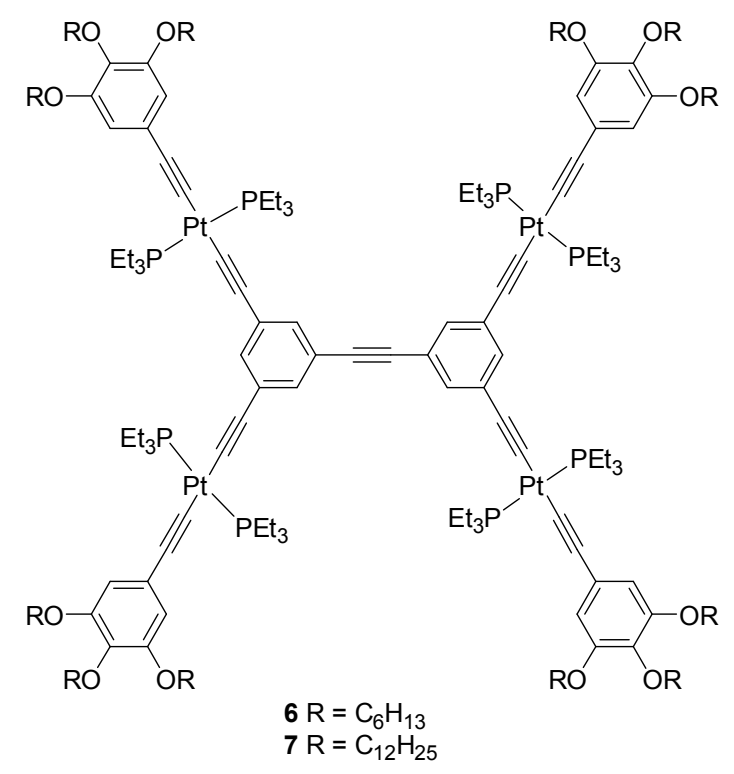

图 3 化合物 6 和 7 的结构

Figure 3 Chemical structures of compounds 6 and 7 

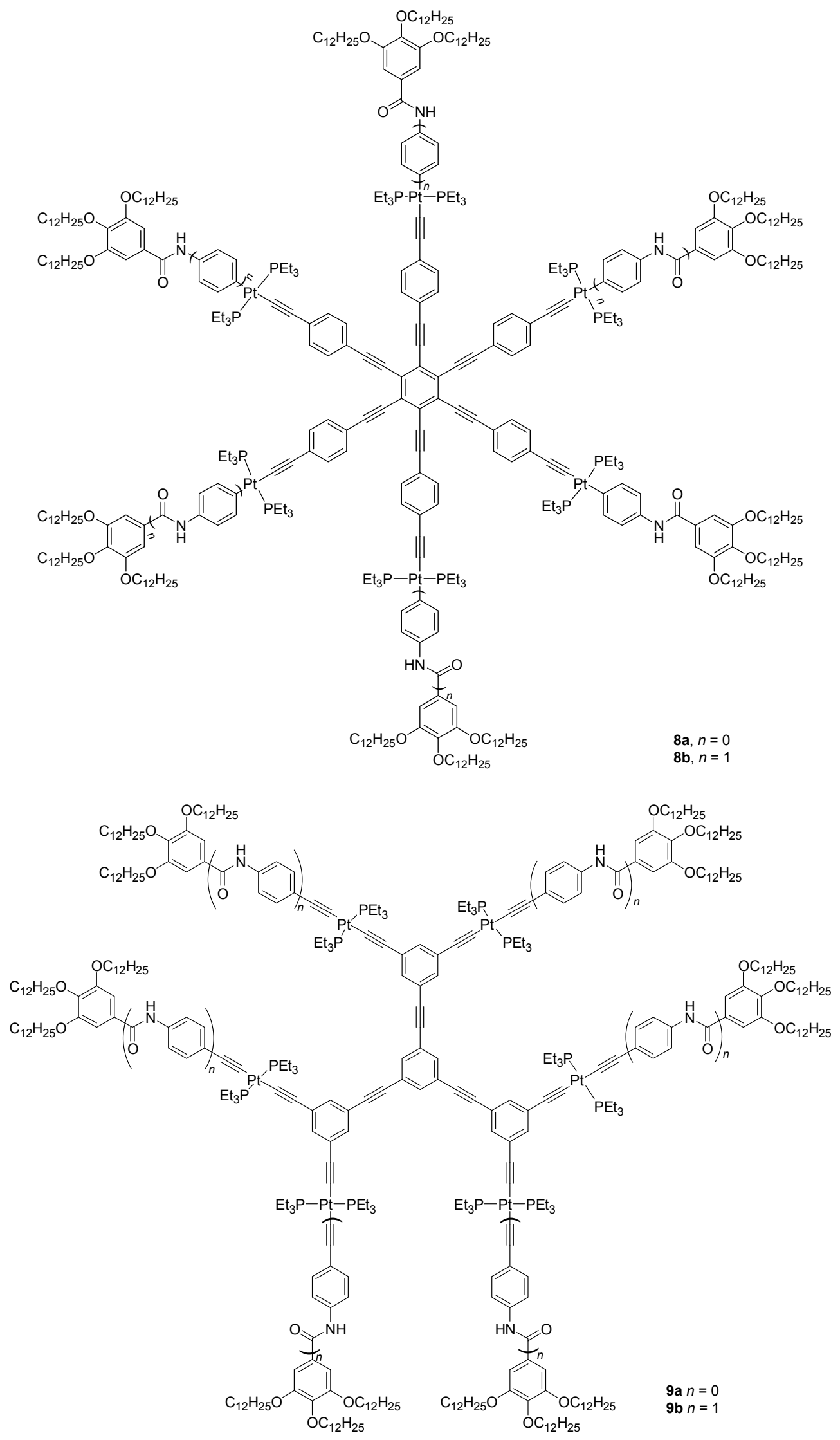

图 4 化合物 $8 a \sim 8 b$ 和 $9 a \sim 9 b$ 的结构

Figure 4 Chemical structures of compounds $8 a \sim 8 b$ and $9 a \sim 9 b$ 
最终功能起着至关重要的作用 ${ }^{[49,50]}$. 利用扫描电子显微 镜(SEM)对这些配合物的形貌进行研究发现, 这些化合 物的聚集形态高度依赖于分子结构. 例如星射状化合物 $8 b$ 可以在混合溶剂(二氯甲烷/丙酮 $=1 / 1$, 体积比)中形 成直径大小在 $0.4 \sim 1.0 \mu \mathrm{m}$ 、类似于纳米小球的形貌，同 时树枝状的化合物 9b 则可以在混合溶剂(DCM/acetone $=3 / 1$, 体积比) 中形成类似的介观形貌, 直径大小在 $0.4 \sim 0.5 \mu \mathrm{m}$. 然而, 没有酰胺基元修饰的化合物 $8 \mathbf{a}$ 和 9a 不能在相同条件下聚集形成规则的纳米结构. 这一 结果表明能够提供氢键作用位点的酰胺基元在化合物 的自聚集过程起到了至关重要的作用. 另外研究发现, 仅有树枝状化合物 9b 能够在一些有机溶剂中形成稳定 的凝胶, 这说明凝胶因子的形状与空间取向对其成胶能 力有很大的影响, 这与之前的研究结果是类似的.

\section{2 官能团化的铂炔衍生物凝胶}

近年来, 通过 “自下而上” 的策略来够构筑功能化 超分子材料得到了人们的广泛关注 ${ }^{[51]}$. 在构筑功能化 有机超分子凝胶体系时，“自下而上” 的构筑策略也被 普遍应用. 通过在凝胶因子中引入一些具有光电活性的 官能团, 则有可能构筑一些具有特殊光电活性与功能的 超分子有机金属凝胶. 例如, 偶氮、萠等结构单元可以 作为修饰凝胶因子的官能团引入到最终的凝胶体系中, 实现对凝胶宏观性质的改良. 同时, 这些功能化的官能 团常常在凝胶相中表现出与单体不同甚至更为优异的 性质. 特别是共轭分子自聚集形成的发光凝胶, 由于其 在场效应晶体管(FET)、有机发光二极管(OLED)、有机 发光晶体管(OLETs)和传感器等方面的潜在应用近年来 备受关注 ${ }^{[52 ~ 54]}$. 最为常见的合成方法即在设计凝胶因 子时引入苂光基团, 例如, Ziessel 课题组 ${ }^{[55]}$ 、George 课 题组 ${ }^{[56,57]}$ 设计并成功开发了一系列发光有机凝胶.

\subsection{1蝶烯修饰的铂炔衍生物超分子金属凝胶}

在过去的几十年, 蝶烯类化合物因其独特的三维结 构, 及其在光电材料、传感器和分子器件等领域的广阔 应用前景, 越来越引起人们的广泛关注, 并在超分子化 学和材料科学中被广泛探索和研究 ${ }^{[58 ~ 62]}$. 然而由于蝶 烯单元的刚性骨架在一定程度上可能会阻碍相应衍生 物的有效聚集, 含蝶烯单元的有机凝胶因子鲜有报道. 2011 年杨海波课题组 ${ }^{[63]}$ 报道了第一例以蝶烯为核心骨 架的超分子有机金属凝胶, 他们设计合成了一系列以三 蝶烯或五蝶烯为核心骨架、铂炔基元桥连的有机金属凝 胶因子(图 5). 研究发现, 这类凝胶因子能够在诸如正己 烷、正戊烷、正辛烷等烷烃类有机溶剂中形成稳定的超 分子有机金属凝胶. 同时通过对环境温度的调控可以实 现溶液相到凝胶相的可逆变化. 其中, 凝胶因子 $10 \mathrm{c}$ 和 11c 可以在正己烷中聚集形成典型的纤维状结构. 进一
步研究发现，改变溶剂极性可以实现对此类凝胶形貌的 调控. 例如，凝胶因子 11c 在极性溶剂(正丙醇)中，聚集 形成小球，与其在非极性溶剂中的纤维状形貌有着明显 的不同. 因此, 这类凝胶的微观形貌可以通过调节溶剂 的极性进行调控.

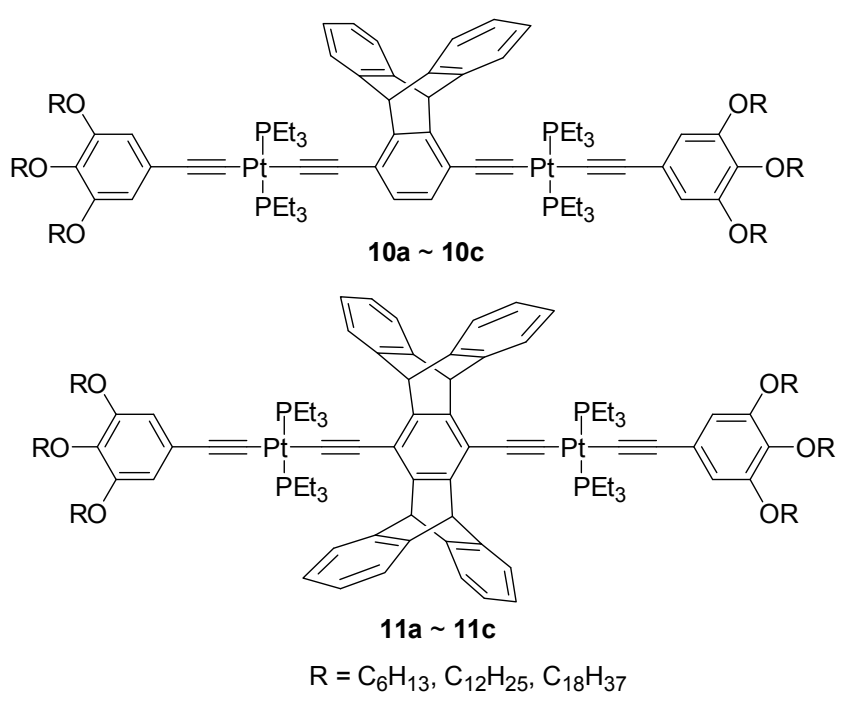

图5 化合物 10a $\sim 10$ 和 $11 \mathrm{a} \sim 11 \mathrm{c}$ 的结构

Figure 5 Chemical structures of compounds 10a $\sim 10 \mathrm{c}$ and $11 \mathrm{a} \sim 11 \mathrm{c}$

\subsection{2 偶氮修饰的铂炔衍生物超分子金属凝胶}

偶氮苯及其衍生物是一类典型的光致异构分子，这 类分子在不同波长的光激发后，可以在两种不同构象之 间发生可逆转换，利用这种性质可以开发出新型的光刺 激响应分子器件 ${ }^{[64]}$. 偶氮苯具有顺反两种异构体, 反式 (trans) 异构体的两个苯环是中心反演对称的, 而顺式 (cis) 异构体的两个苯环在氮氮双键的同一侧，并且是镜 像对称的. 反式构象较顺式构象更稳定, 但在一定频率 范围的光照射下, 反式构象将发生异构化而转化为顺 式.

杨海波课题组 ${ }^{[65]}$ 设计并合成了一系列外围有疏水 长链烷烃修饰、以偶氮基元为核心、铂炔为桥连基元的 铂炔衍生物(图 6). 通过凝胶测试，该课题组发现不含有 酰胺键的化合物 $12 \mathrm{a} \sim 12 \mathrm{c}$ 在常见的极性、非极性、芳 香性溶剂中均不能形成凝胶; 而含有酰胺键化合物 $13 \mathrm{a} \sim 13 \mathrm{c}$ 则能够在许多常见的非极性烷烃溶剂中形成 稳定的凝胶. 外围修饰的长链烷烃提供的疏水作用及酰 胺键提供的氢键作用在凝胶形成过程中起到重要作用. 他们对成胶驱动力及凝胶因子的聚集模式进行了较为 详细深入的研究. 以 $13 \mathrm{~b}$ 分子为例, 对其在正己烷中的 紫外一荣光光谱进行研究发现, 当样品的浓度进行梯度 稀释(从 $2.0 \times 10^{-4} \sim 5.0 \times 10^{-6} \mathrm{~mol} / \mathrm{L}$ ) 后, 其吸收光谱发 生明显变化, 光谱中聚集态的特征峰 $\left(\lambda_{\max }=437 \mathrm{~nm}\right)$ 逐 
渐蓝移到单体吸收的特征峰 $\left(\lambda_{\max }=335 \mathrm{~nm}\right)$, 这一变化 很好地解释了随着浓度的稀释, 聚集态到单体的转变. 在低浓度下主要是以单体形式存在, 而在高浓度下则主 要以聚集体形式存在, 并且是以 $\mathrm{J}$ 型聚集模式存在的. 通过对模型 12'(图 7)的单晶结构分析, 观测到溶剂分子 $N, N$-二甲基甲酰胺(DMF)与分子之间的氢键作用. 为了 进一步阐述氢键在该类化合物成胶过程中的重要作用, 该课题组进一步进行了研究, 首先利用化合物 $13 \mathrm{~b}$ 在正 己烷中形成稳定凝胶, 再向其中缓慢滴加极性溶剂 DMF, 稳定的凝胶相发生了坍塌, 这一宏观状态的变化 可以证明, DMF 的加入会破坏分子间的氢键, 进而导致 超分子有机金属凝胶的解体, 从而进一步证明了酰胺键 所提供的氢键是成胶的重要推动力.

通过对光谱的研究发现, 化合物 12a 12b 和 13a $13 \mathrm{c}$ 在溶液相中都具有光刺激响应性, 在紫外光光照条 件下, 部分反式偶氮转化为顺式结构. 然而较为遗憾的 是, 由这类偶氮基元修饰的中性铂炔基元所构筑的超分 子有机金属凝胶并不具备光刺激响应性. 但进一步研究 表明, 该系列铂炔凝胶因子所形成的超分子有机金属凝 胶的介观形貌呈现为十分规整的蜂窝状结构. 这与传统
的纤维状结构存在很大的差异，这也是首例基于中性铂 炔基元呈蜂窝状结构的超分子有机金属凝胶.

\subsection{3 萠修饰的铂炔衍生物超分子金属凝胶}

萠作为一类常见的 $\pi$-共轭发色官能团, 已经被广泛 应用于发光凝胶的制备和研究中. 例如, Kato 课题组 ${ }^{[66]}$ 在 2007 年报道了一例利用萠基元修饰的树枝状凝胶因 子, 进而成功构筑了一类颜色可调控的蓝色苂光凝胶. 不少课题组已经证明了萠衍生物的发射波长是可以调 节的，例如通过引入乙炔基实现对 $\pi$-共轭体系的扩展， 实现发射波长从紫外区域到可见区域的延伸 ${ }^{[67]}$. 随着 这一合成策略的成功实现，乙炔基萠衍生物及其低聚物 已在光电器件和生物探针等方面引起了人们广泛兴 趣 ${ }^{[68,69]}$. 杨海波课题组 ${ }^{[70]}$ 通过萠结构单元与铂炔基元的 结合，成功构筑了具有发光性质的以乙炔基萠为核心的 超分子有机金属凝胶(图 8).

通过中心萠基团的 $\pi-\pi$ 堆积作用和外围长链烷烃的 疏水作用及分子间氢键作用，该类新型铂炔衍生物在正 己烷、环已烷、正丙醇等中均形成稳定的凝胶. 与之前 报道的工作类似，氢键的存在对凝胶因子的成胶性能具 有重要的影响. 例如, 通过对含有和不含有酰胺键的两

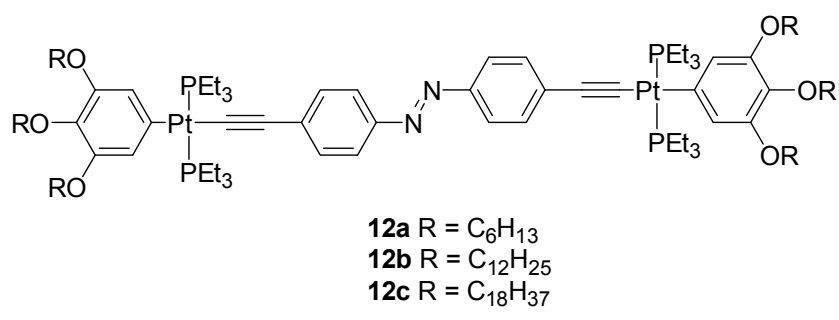

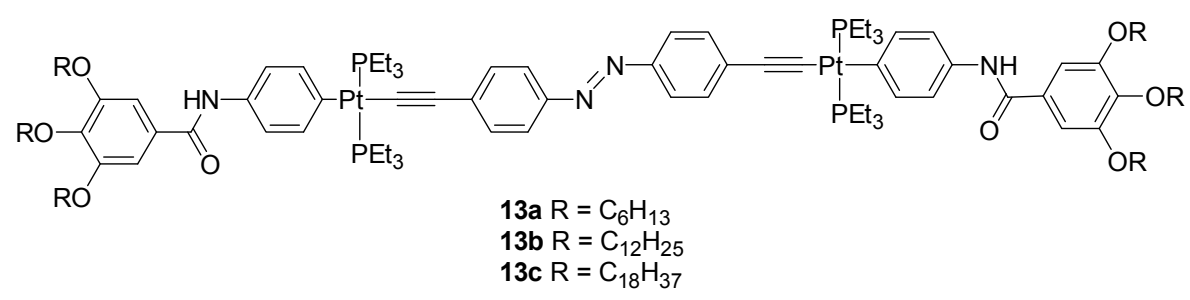

图 6 化合物 12a $\sim 12 \mathrm{c}$ 和 13a $\sim 13 \mathrm{c}$ 的结构

Figure 6 Chemical structures of compounds 12a $\sim 12 \mathrm{c}$ and 13a $\sim 13 \mathrm{c}$

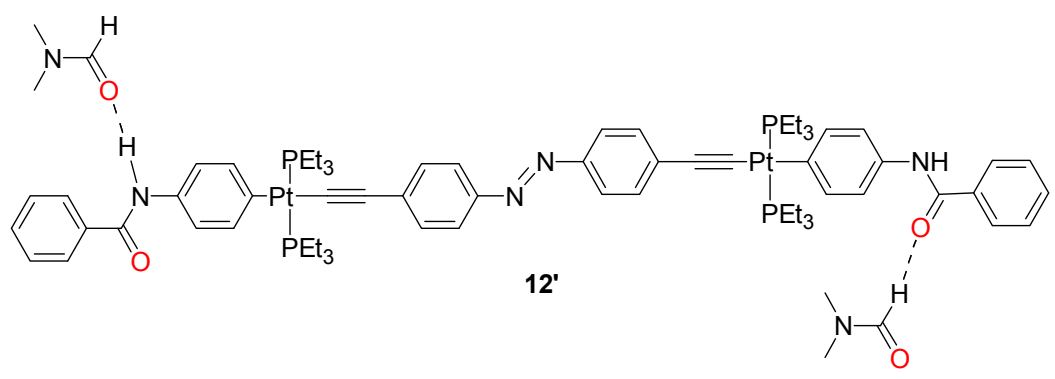

图 7 化合物 $\mathbf{1 2}$ '的结构

Figure 7 Chemical structure of compound 12' 
<smiles>[R]C1=CC([R])=C2C=CC3=C([R])C=C([R])C4=CC=C1C2C43</smiles><smiles>CCOc1cc(C(=O)Cc2ccc(C(CC)(CC)PI)cc2)cc(OCC)c1OCC</smiles>
$n=1, \mathrm{~B}$
$R^{1}=A, R^{2}=R^{3}=R^{4}=H$ 14a; $R^{1}=B, R^{2}=R^{3}=R^{4}=H$ 14b

$R^{1}=R^{3}=A, R^{2}=R^{4}=H 15 a ; \quad R^{1}=R^{3}=B, R^{2}=R^{4}=H 15 b$

$R^{1}=R^{4}=A, R^{2}=R^{3}=H 16 a ; \quad R^{1}=R^{4}=B, R^{2}=R^{3}=H 16 b$

$R^{1}=R^{2}=R^{3}=R^{4}=A$ 17a; $\quad R^{1}=R^{2}=R^{3}=R^{4}=B$ 17b

图 8 化合物 14a 14b, 15a 15b, 16a 16b 和 17a $\sim 17 b$ 的结构

Figure 8 Chemical structures of compounds 14a $\sim 14 b, 15 a \sim 15 b, 16 a \sim 16 b$ and $17 a \sim 17 b$

类化合物的成胶性能进行比较发现, 酰胺键的引入有利 于分子间氢键的形成, 进而驱动其形成稳定的凝胶. 有 趣的是, 通过对该类化合物的光谱分析发现, 随着共轭 铂乙炔片段的引入不仅可以调节分子的尺寸大小, 还可 以有效调整萠结构单元的发射性质, 使其从紫外光区域 红移到长波长区域. 例如, 化合物 $\mathbf{1 7 a}$ 与不含任何修饰 基团的萠相比, 前者的吸收光谱主要吸收带在 458 $490 \mathrm{~nm}$, 而后者仅为 $335 \mathrm{~nm}$, 化合物 17a 的吸收峰发生 明显的红移. 此外, 每一个化合物的发射光谱与最低能 量的吸收峰呈镜像对称, 这表明了吸收和发射过程中具 有相同的光学跃迁. 随着引入的铂乙炔基团数的增加, 这类以萠为中心、铂乙炔基元为桥连的凝胶因子的吸收 和发射的最大值可以迁移到长波长区域, 并且其摩尔吸 光系数大大提高. 此外, 溶液中超分子聚集体的浓度或 温度依赖吸收光谱和发射光谱研究进一步表明, 铂炔基 元可以调整它们的发光性质, 使其发光从蓝色迁移到黄 色. 此外, 所形成的超分子有机金属凝胶也表现出较强 的苂光性能, 并且其荧光发射波长可通过凝胶因子的结 构改变进行调节. 同样, 利用 SEM 可以更好地研究其凝 胶态的形貌，了解其介观自聚集行为. 对比这一系列化 合物所形成的干凝胶形貌可以发现, 随着萠外围长链烷 烃修饰基团的增多及烷基链链长的增加, 分子间的交联 程度明显增加.

萠结构基元也可以作为外围修饰基元对中性铂炔 衍生物进行官能团化, 此时作为功能性基元的萠模块位 于分子外围, 有可能更有利于展示萠基元的功能. 据报 道, 芳香性的萠结构基元可与石墨烯共轭体系发生 $\pi-\pi$ 相互作用 ${ }^{[71,72]}$. 基于此, 2014 年, 杨海波课题组 ${ }^{[73]}$ 设计 合成了一系列外围萠修饰的铂炔衍生物用于构筑超分 子有机金属凝胶, 并将其进一步应用于石墨烯的分散 中, 该课题组通过偶联、醚化和酰胺化等几步反应, 成 功构筑了两类含有不同萠数目的凝胶因子(图 9).

研究发现, 这类外围有萠修饰的铂炔衍生物与芘以 内核形式修饰的铂炔衍生物相比, 其成胶条件更为复 杂, 前者需在混合溶剂之中才能形成稳定的凝胶. 芳环 外围修饰的疏水长链提供的疏水作用、酰胺键提供的氢 键作用以及 $\pi-\pi$ 堆积等弱相互作用均为 $18 \mathrm{a} \sim 18 \mathrm{~b}$ 和
$19 a \sim 19 b$ 成胶的主要驱动力. 通过 $18 a \sim 18 b$ 和 19a $19 \mathrm{~b}$ 的成胶测试对比发现, 相比 18a $\sim 18 \mathrm{~b}$, 改性的凝胶 因子 19a 19b 在成胶时需要更多的芳香类溶剂. 例如, 19a 需要在正己烷和苯以 $5: 2$ 的比例混合的溶液中才 能形成凝胶, 而 $18 \mathrm{a}$ 则在正己烷和苯以 $5: 1$ 比例混合的 溶剂中就能形成稳定的凝胶. 这类萠修饰的超分子有机 金属凝胶在一定的温度范围内, 可以实现凝胶相-溶液 相之间的可逆转变.

基于外围萠修饰的铂炔衍生物构筑的超分子有机 金属凝胶可在一定波长激发下发出蓝色荧光, 有意思的 是，在凝胶中掺杂一定量的石墨烯之后，其荧光发射强 度明显降低. 这是由于萠基元与石墨烯发生了电荷转移 相互作用. 利用透射电子显微镜(TEM)对其介观聚集行 为进行研究时发现, 石墨烯片状结构较为均匀地分散于 交联的纤维之中, 且对原凝胶的形貌没有明显的影响与 破坏. 对混合凝胶的流变性能进行研究发现, 相较于单 一组分的凝胶, 混合凝胶的储存模量和损耗模量均有明 显增加, 说明分散了石墨烯的混合凝胶比原单一组分凝 胶更具有刚性. 这也是首例将铂炔有机金属凝胶应用于 石墨烯分散的例子.

\section{2 结论}

通过以上介绍可以看出, 近年来, 基于中性铂炔基 元的超分子有机金属凝胶的研究不断深入, 有了长足的 发展. 利用线性铂炔基元作为桥连结构单元, 可以构筑 一系列中性的凝胶因子, 并通过引入不同官能团, 例如 具有光电活性的偶氮、萠基元等, 可以实现对凝胶因子 的改性. 同时这些基于铂炔基元的有机金属凝胶也通过 多重弱相互作用的协同效应在大尺度上表现出许多不 同于单体的宏观性质. 另一方面，虽然目前对基于中性 铂炔基元的超分子有机金属凝胶已有课题组进行了较 为深入的研究, 同时也成功构筑了一系列具有结构性质 易调控等优良特点的凝胶, 但其研究仍然还处于较为基 础的阶段, 研究内容主要停留在凝胶因子的设计与合成 上，对其所形成的有机金属凝胶的性质与应用的研究还 不够深入. 因此，随着超分子有机金属凝胶领域的发展， 继续拓展和深入研究基于中性铂炔基元的超分子有机 

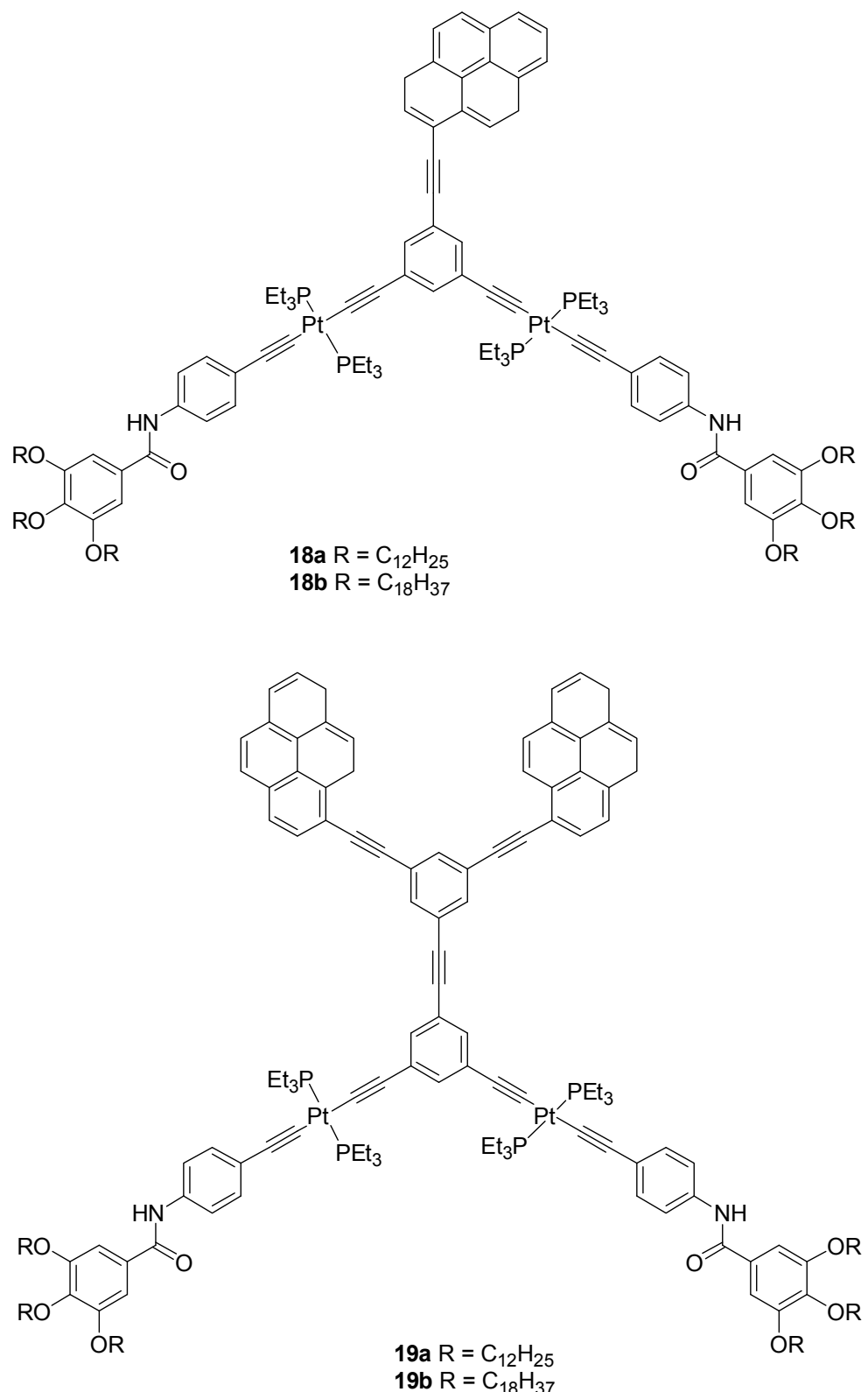

图 9 化合物 18a $\sim 18 b, 19 a \sim 19 b$

Figure 9 Chemical structures of compounds $18 a \sim 18 b, 19 a \sim 19 b$

金属凝胶及其在功能材料领域的应用是十分必要的.

\section{References}

[1] Flory, P. J.; Faraday, D. Chem. Soc. 1974, $57,7$.

[2] Terech P; Weiss, R. G. Adv. Mater. 2000, 12, 1237.

[3] Lin, Y.-C.; Weiss, R. G. Macromolecules 1987, 20, 414.

[4] Hoeben, F. J. M.; Jonkhejim, P.; Meijer, E. W.; Schenning, A. P. H. Chem. Rev., 2005, 105, 1491.

[5] Fages, F. Angew. Chem., Int. Ed. 2006, 45, 1680.

[6] Sangeetha, N. M.; Maitra, U. Chem. Soc. Rev., 2005, 34, 821.

[7] Jang, W. D.; Jiang, D.-L.; Aida, T. J. Am. Chem. Soc. 2000, 122, 3232 .

[8] Kawano, S.; Fujita, N.; Bommel, K. J. C.; Shinkai, S. Chem. Lett. 2003, 32, 12 .

[9] George, S. J.; Ajayaghosh, A.; Jonkeijm, P.; Schenning, A. P. H. J.;
Meijer, E. W. Angew. Chem., Int. Ed. 2004, 43, 3422.

[10] Dzolic, Z.; Cametti, M.; Cort, A. D. Chem. Commun. 2007, 3535.

[11] Liu, Q.; Wang, Y.; Li, W.; Wu, L. Langmuir 2007, 23, 8217.

[12] Stanley, C. E.; Clarke, N.; Anderson, K. M. Chem. Commun. 2006, 3199.

[13] Maeda, H.; Haketa, Y.; Nakanishi, T. J. Am. Chem. Soc. 2007, 129, 13661.

[14] Klawonn, T.; Gansauer, A.; Winkler, I. Chem. Commun. 2007, 1894.

[15] Tam, A. Y. Y.; Wong, K. M. C.; Wang, G.-X. Chem. Commun. 2007, 2028.

[16] Sangeetha, N. M.; Maitra, U. Chem. Soc. Rev. 2005, 34, 821.

[17] Wang, Y. J.; Tang, L. M.; Yu, J. Prog. Chem. 2009, 21, 1312.

[18] Anthony, Y. Y.; Yam, V. W. W. Chem. Soc. Rev. 2013, 42, 1540.

[19] Xing, B.; Choi, M. F.; Xu, B. Chem. Commun. 2002, 362.

[20] Xing, B.; Choi, M. F.; Zhou, Z.; Xu, B. Langmuir 2002, 18, 9654. 
[21] Xing, B.; Choi, M. F.; Xu, B. Chem. Eur. J. 2002, 8, 5028.

[22] Cho, Y.; Lee, J. H.; Jaworski, J.; Park, S.; Lee, S. S.; Jung, J. H. New J. Chem. 2012, 36, 32.

[23] Yamada, Y. M. A.; Maeda, Y.; Uozumi, Y.; Org. Lett. 2006, 8, 4259.

[24] Kishimura, A.; Yamashita, T.; Aida, T. J. Am. Chem. Soc. 2005, $127,179$.

[25] Marpu, S.; Hu, Z.; Omary, M. A. Langmuir 2010, 26, 15523.

[26] Pettijohn, C. B.; Jochnowitz, E. B.; Chuong, B.; Nagle, J. K.; Vogler, A. Coord. Chem. Rev. 1998, 171, 85.

[27] McMillin, D. R.; Moore, J. J. Coord. Chem. Rev. 2002, 229, 113.

[28] Gareth Williams, J. A. Top. Curr. Chem. 2007, 281, 205.

[29] Wong, K. M. C.; Yam, V. W. W. Coord. Chem. Rev. 2007, 251, 2477.

[30] Eryazici, I.; Moorefield, C. N.; Newkome, G. R. Chem. Rev. 2008, $108,1834$.

[31] Wong, K. M. C.; Yam, V. W. W. Acc. Chem. Res. 2011, 44, 424.

[32] Wong, K. M. C.; Yam, V. W. W. Chem. Commun. 2011, 47, 11579.

[33] Tam, A. Y. Y.; Wong, K. M. C.; Yam, V. W. W. J. Am. Chem. Soc. 2009, 131, 6253.

[34] Li, Y.-G.; Tam, A. Y. Y.; Wong, K. M. C.; Wu, W. Li, L.; Yam, V. W. W. Chem. Eur. J. 2011, 17, 8048.

[35] Tam, A. Y. Y.; Wong, K. M. C.; Wang, G. X.; Yam, V. W. W. Langmuir 2009, 25, 8685 .

[36] Camerel, F.; Ziessel, R.; Donnio, B.; Bourgogne, C.; Guillon, D.; Schmutz, M.; Iacovita, C.; Bucher, J. P. Angew. Chem., Int. Ed. 2007, 46, 2659.

[37] Wang, W.; Yang, H.-B.; Chem. Commun. 2014, 50, 5171-5186.

[38] Cardolaccia, T.; Li, Y. J.; Schanze, K. S. J. Am. Chem. Soc. 2008, $130,2535$.

[39] Beljonne, D.; Wittmann, H. F.; Kohler, A.; Graham, S.; Younus, M.; Lewis, J.; Raithby, P. R.; Khan, M. S.; Friend, R. H.; Bredas, J.-L. J. Chem. Phys. 1996, 105, 3868.

[40] Liu, Y.; Jiang, S.; Glusac, K.; Powell, D. H.; Anderson, D. F.; Schanze, K. S. J. Am. Chem. Soc. 2002, 124, 12412.

[41] Haskins-Glusac, K.; Pinto, M. R.; Tan, C. Y.; Schanze, K. S. J. Am. Chem. Soc. 2004, 126, 14964.

[42] Tian, Y.-J.; Meijer, E. W.; Wang, F.; Chem. Commun. 2013, 49, 9197.

[43] Chen, L.-J.; Zhang, J.; He, J.-M.; Xu, X.-D.; Wu, N.-W.; Wang, D.-X.; Abliz, Z.; Yang, H.-B. Organometallics 2011, 30, 5590.

[44] Llusar, M.; Sanchez, C. Chem. Mater. 2008, $20,782$.

[45] Xia, Y.; Wang, Y.; Chen, K.; Tang, L. M. Chem. Commun. 2008, 5113.

[46] Han, M. G.; Foulger, S. H.; Adv. Mater. 2004, 16, 226.

[47] Jiang, B.; Chen, L.-J.; Xu, L.; Liu, S.-Y.; Yang, H.-B. Chem. Commun. 2013, 49, 6977.
[48] Mohnani, G. S.; Bonifazi, D. Coord. Chem. Rev. 2010, 254, 2342.

[49] Xue, M.; Yang, Y.; Chi, X.; Zhang, Z.; Huang, F. Acc. Chem. Res. 2012, 45, 1294.

[50] Meazza, L.; Foster, J. A.; Fucke, K.; Metrangolo, P.; Resnati, G.; Steed, J. W. Nat. Chem. 2012, 4, 437.

[51] Liu, H.; Xu, J.; Li, Y. Acc. Chem. Res. 2010, 43, 1496.

[52] Tu, T.; Assenmacher, W.; Peterlik, H.; Weisbarth, R.; Nieger, M.; Dçtz, K. H. Angew. Chem., Int. Ed. 2007, 46, 6368.

[53] Hoeben, F. J. M.; Jonkheijm, P.; Meijer, E. W.; Schenning, A. P. H. J. Chem. Rev. 2005, 105, 1491.

[54] Ajayaghosh, A.; Praveen, V. K. Acc. Chem. Res. 2007, 40, 644.

[55] Camerel, F.; Ziessel, R.; Donnio, B.; Bourgogne, C.; Guillon, D.; Schmutz, M.; Iacovita, C.; Bucher, J. P. Angew. Chem., Int. Ed. 2007, 46, 2659.

[56] Ajayaghosh, A.; George, S. J. J. Am. Chem. Soc. 2001, 123, 5148.

[57] George, S. J.; Ajayaghosh, A.; Jonkheijm, P.; Schenning, A. P. H. J. Meijer, E. W. Angew. Chem., Int. Ed. 2004, 43, 3422.

[58] Chong, J. H.; MacLachlan, M. J. Chem. Soc. Rev. 2009, 38, 3301.

[59] Yang, J.-S.; Yan, J.-L.; Hwang, C.-Y.; Chiou, S.-Y.; Liau, K.-L.; Tsai, H.-H.; Lee, G.-H.; Peng, S.-M. J. Am. Chem. Soc. 2006, 128, 14109.

[60] Chen, C.-F. Chem. Commun. 2011, 47, 1674.

[61] Yang, J.-S.; Huang, Y.-T.; Ho, J.-T.; Sun, W.-T.; Huang, H.-H.; Lin, Y.-C.; Huang, S.-J.; Huang, S.-L.; Lu, H.-F.; Chao, I. Org. Lett. 2008, 10, 2279.

[62] Zhu, X.-Z.; Chen, C.-F. J. Am. Chem. Soc. 2005, 127, 13158.

[63] Zhang, J.; Xu, X.-D.; Chen, L.-J.; Luo, Q.; Wu, N.-W.; Wang, D.-X.; Zhao, X.-L.; Yang, H.-B. Organometallics 2011, 30, 4032.

[64] Delaire, J. A.; Nakatanik, K. Chem. Rev. 2000, 100, 15.

[65] Xu, X.-D.; Zhang, J.; Chen, L.-J.; Zhao, X.-L.; Wang, D.-X.; Yang, H.-B. Chem. Eur. J. 2012, 18, 1659.

[66] Kamikawa, Y.; Kato, T. Langmuir 2007, 23, 274.

[67] Maeda, H.; Maeda, T.; Mizuno, K.; Fujimoto, K.; Shimizu, H.; Inouye, M. Chem. Eur. J. 2006, 12, 824.

[68] Zhao, Q.; Yu, M.-X..; Shi, L.-X., Liu, S.-J., Li, C.-Y., Shi, M., Zhou, Z.-G., Huang, C.-H.; Li, F.-Y. Organometallics 2010, 29, 1085.

[69] Yang, Y.-M.; Zhao, Q.; Feng, W.; Li, F.-Y. Chem. Rev. 2012, 113, 192.

[70] Xu, X.-D.; Zhang, J.; Yu, X.-D.; Chen, L.-J.; Wang, D.-X.; Yi, T.; Li, F.-Y.; Yang, H.-B. Chem.-Eur. J. 2012, 18, 16000.

[71] Guo, S..; Dong, S.-J. Chem. Soc. Rev. 2011, 40, 2644.

[72] Yu, G.; Xue, M.; Zhang, Z.; Li, J.; Han, C.; Huang, F. J. Am. Chem. Soc. 2012, 134, 13248.

[73] Wu, N.-W.; Zhang, J.; Xu, X.-D.; Yang, H.-B. Chem. Commun. 2014, 50, 10269. 